\title{
Study on the effect of home night lighting environment on students' eyestrain and visual efficacy
}

\author{
J.Q. Wang ${ }^{1}$, Y.H. Fang ${ }^{2}$, Y.X. Huang ${ }^{2}$, J.N. Chen ${ }^{2}$ and X.Y. Luo ${ }^{2 *}$ \\ ${ }^{1}$ Hangzhou Education Development Service Center, Hangzhou, Zhejiang, CHINA \\ ${ }^{2}$ College of Civil Engineering and Architecture, Zhejiang University, Hangzhou, Zhejiang, CHINA
}

\begin{abstract}
China has the highest incidence rate of myopia, and the number of teenagers with myopia accounts for more than half of the total number of myopia in the world. In recent years, With the increase of youth night home learning time, the impact of the night lighting environment in the home learning space on eye health has become increasingly obvious. This paper selects 112 home learning spaces of middle school students to carry out the actual measurement of lighting environment parameters and the satisfaction survey of the lighting environment, and through comparative experiments to explore the effects of illuminance, color temperature and lighting methods on eyestrain and visual efficacy. This Study found that under mixed lighting conditions, when the color temperature is $4500 \mathrm{k}$, the illuminance in the range of 5001x-7501x is beneficial to relieve eyestrain and improve visual efficacy; When the illuminance is $5001 \mathrm{x}$, a low color temperature (3500k) can help relieve eyestrain, and a high color temperature (6000k) can improve visual efficacy better. In addition, mixed lighting is better than local lighting for relieving eyestrain and improving visual efficacy. The results will help improve the night lighting environment in the home learning space of middle school students.
\end{abstract}

\section{Introduction}

Approximately 2.2 billion people worldwide suffer from some form of visual impairment [1]. Especially in East Asia, myopia has become a major health problem among teenager [2]. China is the country with the highest incidence rate of myopia [3], with 600 million patients with myopia. In 2018, the number of teenagers with myopia in China accounted for $53.6 \%$ of the total number of myopia in the world, while the proportion of teenager with myopia in other countries during the same period was much lower than that of China [4]. The increase in the incidence rate of myopia is a problem that deserves worldwide attention.

This study found that eye health problems are not only related to age and habits with the eyes, but also closely related to the amount of schoolwork and time with eyes. In addition, a very important factor that causes myopia is the lighting environment [5]. The lighting environment will not only bring the eye health threats, such as refractive error (myopia, hyperopia), retinal damage, etc., but also harms the physiological functions of the central nervous system of the human body, and affects the circadian rhythm. At the same time, the lighting environment will also have a certain impact on people's learning ability, visual efficacy, and psychological emotions [6].

At present, some scholars have chosen school buildings as subjects to study the influence of lighting environment parameters on students. De Giuli et al. [7] tested the lighting environment of 7 primary schools, meanwhile, conducted a survey on 614 students in these primary schools, and found that the illuminance and uniformity of illuminance will have a certain impact on the learning efficiency and comfort of students. The two research groups Zadnik [8] and Morgan [9] focused on primary students and classrooms, and found that the mean visual acuity of primary students is proportional to the illuminance of the desktop, and primary students are more sensitive to and more affected by lighting environment. Shimomura et al. [10] explored the effect of color temperature on attention during learning through experiments, and found that the drowsiness at low color temperature is more significant than that at high color temperature, and high color temperature is conducive to concentration. Through experimental comparison, Inoue [11] found that eyestrain is easy to occur at high color temperature $(6700 \mathrm{k})$.

However, the subjects of the above studies are all the classroom lighting environment, with background lighting as the main lighting method. However, after investigation, we found that $22.29 \%$ of students had a mean learning time of more than 3 hours after returning home, and $64.69 \%$ had a mean learning time of 1 hour to 3 hours. Therefore, the lighting environment of the home learning space is also particularly important for students' eye health. Home learning spaces are different from classrooms, and most of them use mixed lighting (local lighting integrate with background lighting) method, and there are few related studies at present.

First of all, this study selected the learning space of 112 families to test the lighting environment parameters and 
issue the subjective questionnaire. Secondly, the relationship between lighting environment parameters and satisfaction is studied. Finally, experiments were carried out on the night lighting environment of middle school students' learning space to explore the effect of illuminance, color temperature and lighting methods on eyestrain and visual efficacy. The results of this studies are helpful to provide a reference for the design and improvement of night lighting environment in the learning space of middle school students, and to improve people's awareness of healthy lighting.

\section{Methods}

\subsection{Investigation of home lighting environment at night}

\subsubsection{Data measured}

In this paper, the current situation of night lighting environment in residential learning space of 112 middle school students was surveyed. In order to avoid the interference of natural lighting, nighttime learning time was selected for testing by means of data measurement. In the test, the background lighting was fully turned on, and the desk lamp was set to the user's common mode. In order to describe the lighting environment of the learning space, SFIM-400 spectral strobe illuminometer was used to test the illuminance, color temperature, strobe of lamps and color rendering index on the working surface.

The basic situation of the survey objects is shown in Table.1, it is found that most of the desks are placed in the corner of the room, which causes the background lighting to have little effect on the illuminance of the working surface. In addition, $12.5 \%$ of the desk lamps cannot be adjusted on demand, resulting in a single lighting environment. And $7.14 \%$ of the desk lamps are placed on the right hand side or in the middle, causing part of the working surface to be shaded or over-illuminated. Beyond that, the lighting environment level varies greatly. Among the 112 sets of data tested, the lowest illuminance is 109.381x, and the highest illuminance is as high as 2145.011x. In terms of color temperature, most students prefer cool white light sources when studying with desk lamps. Only a few use warm yellow light sources, and the color temperature of the light source is generally high.

Table.1 Basic situation

\begin{tabular}{|c|c|c|}
\hline \multicolumn{2}{|c|}{ Category } & Proportion \\
\hline \multirow[t]{2}{*}{ Gender } & Male & $42 \%$ \\
\hline & Female & $58 \%$ \\
\hline \multirow[t]{3}{*}{ Age } & $\leq 12$ & $2.23 \%$ \\
\hline & $13-18$ & $93.68 \%$ \\
\hline & $\geq 18$ & $4.09 \%$ \\
\hline \multirow[t]{4}{*}{ Vision } & $\geq 5.0$ & $16.07 \%$ \\
\hline & $4.9-5.0$ & $20.53 \%$ \\
\hline & $4.6-4.8$ & $26.78 \%$ \\
\hline & $\leq 4.5$ & $36.60 \%$ \\
\hline \multirow{4}{*}{$\begin{array}{l}\text { Learning time } \\
\text { at night }\end{array}$} & $<1 \mathrm{~h}$ & $12.83 \%$ \\
\hline & $1-2 \mathrm{~h}$ & $36.62 \%$ \\
\hline & $2-3 h$ & $28.07 \%$ \\
\hline & $>3 \mathrm{~h}$ & $22.49 \%$ \\
\hline \multirow{2}{*}{$\begin{array}{c}\text { The location } \\
\text { of learning } \\
\text { space }\end{array}$} & Living room & $8.04 \%$ \\
\hline & Bedroom & $91.96 \%$ \\
\hline \multirow{2}{*}{$\begin{array}{l}\text { Whether the } \\
\text { desk lamp is } \\
\text { adjusted on } \\
\text { demand }\end{array}$} & Can & $87.5 \%$ \\
\hline & Can't & $12.5 \%$ \\
\hline \multirow{3}{*}{$\begin{array}{c}\text { Lamp } \\
\text { placement }\end{array}$} & Left hand side & $92.86 \%$ \\
\hline & Right hand side & $2.68 \%$ \\
\hline & The middle & $4.46 \%$ \\
\hline
\end{tabular}

\subsubsection{Satisfaction survey}

This study carried out a satisfaction survey for middle school students in learning spaces with local lighting environment at night, and the subject of the survey is the users in the measured samples above. The survey time is between April 2020 and May 2020. To avoid the influence of natural lighting, it will be conducted after dark. Questionnaires were distributed during the testing process to facilitate one-to-one correspondence between subjective results and objective test data. A total of 112 questionnaires were distributed and 112 valid questionnaires were returned.

Students' satisfaction with various lighting parameters in the learning space is shown in Table.2.

Table.2 Satisfaction of the lighting parameters of the student's home study space

\begin{tabular}{|c|c|c|c|c|c|c|c|}
\hline Category & \multicolumn{9}{|c|}{ Proportion } \\
\hline \multirow{2}{*}{ Illuminance } & $\begin{array}{c}\text { Extremely } \\
\text { dissatisfied }\end{array}$ & Dissatisfied & $\begin{array}{c}\text { Slightly } \\
\text { dissatisfied }\end{array}$ & $\begin{array}{c}\text { Just } \\
\text { satisfied }\end{array}$ & $\begin{array}{c}\text { Slightly } \\
\text { satisfied }\end{array}$ & Satisfied & $\begin{array}{c}\text { Extremely } \\
\text { satisfied }\end{array}$ \\
\cline { 2 - 8 } & $13.46 \%$ & $8.65 \%$ & $16.35 \%$ & $38.46 \%$ & $10.58 \%$ & $7.69 \%$ & $4.81 \%$ \\
\hline $\begin{array}{c}\text { Illumination } \\
\text { uniformity }\end{array}$ & $\begin{array}{c}\text { Extremely } \\
\text { dissatisfied }\end{array}$ & Dissatisfied & $\begin{array}{c}\text { Slightly } \\
\text { dissatisfied }\end{array}$ & $\begin{array}{c}\text { Just } \\
\text { satisfied }\end{array}$ & $\begin{array}{c}\text { Slightly } \\
\text { satisfied }\end{array}$ & Satisfied & $\begin{array}{c}\text { Extremely } \\
\text { satisfied }\end{array}$ \\
\cline { 2 - 8 } & $1.79 \%$ & $2.68 \%$ & $7.14 \%$ & $33.93 \%$ & $23.21 \%$ & $21.43 \%$ & $9.82 \%$ \\
\hline $\begin{array}{c}\text { Color } \\
\text { temperature }\end{array}$ & $\begin{array}{c}\text { Extremely } \\
\text { dissatisfied }\end{array}$ & Dissatisfied & $\begin{array}{c}\text { Slightly } \\
\text { dissatisfied }\end{array}$ & $\begin{array}{c}\text { Just } \\
\text { satisfied }\end{array}$ & $\begin{array}{c}\text { Slightly } \\
\text { satisfied }\end{array}$ & Satisfied & $\begin{array}{c}\text { Extremely } \\
\text { satisfied }\end{array}$ \\
\cline { 2 - 8 } & $1.79 \%$ & $5.36 \%$ & $8.93 \%$ & $27.68 \%$ & $21.43 \%$ & $19.64 \%$ & $15.18 \%$ \\
\hline \multirow{2}{*}{$\begin{array}{c}\text { Overall } \\
\text { assessment }\end{array}$} & $\begin{array}{c}\text { Extremely } \\
\text { dissatisfied }\end{array}$ & Dissatisfied & $\begin{array}{c}\text { Slightly } \\
\text { dissatisfied }\end{array}$ & $\begin{array}{c}\text { Just } \\
\text { satisfied }\end{array}$ & $\begin{array}{c}\text { Slightly } \\
\text { satisfied }\end{array}$ & Satisfied & $\begin{array}{c}\text { Extremely } \\
\text { satisfied }\end{array}$ \\
\cline { 2 - 8 } & $2.03 \%$ & $1.12 \%$ & $4.28 \%$ & $42.01 \%$ & $29.87 \%$ & $16.45 \%$ & $4.24 \%$ \\
\hline
\end{tabular}


Overall, $92.57 \%$ of students are satisfied with the current lighting environment. Among them, the satisfaction of illuminance uniformity is the best, followed by color temperature. Satisfaction with illuminance is relatively low. $38.55 \%$ of students are dissatisfied with illuminance, and $13.46 \%$ are very dissatisfied. The reason may be that some students mistakenly believe that higher illuminance is more beneficial to eye health, which directly leads to uncomfortable feelings caused by inappropriate illuminance, thereby reducing satisfaction.

\subsection{Eyestrain and visual efficacy experiment}

In order to further explore the impact of the lighting environment in the home learning space of middle school students on students' eyestrain and visual efficacy, this study simulates the learning space in students' home to build a laboratory (Fig.1), and conducts experiments by setting up different lighting environments.

\subsubsection{Experiment Overview}

A total of 100 middle school students, aged between 13 and 18, were selected for the experiment. The ratio of male to female was close to $1: 1$, including 46 boys and 54 girls. The visual acuity of all subjects was above 5.0 after visual correction. All of them had normal color vision, healthy body, regular life and rest, and had no eye diseases und no bad behaviors, like alcohol, smoking, drinking coffee, drugs etc., and did not participate in other clinical experiments.

The experiment is conducted in a teaching office in a middle school. The walls of the office are all white paint, and the desktop is made of wood. The reflectivity of the wall and desktop meets the requirements of "Architectural Lighting Design Standard" GB 50034-2013[12]. In the process of entrance test, it was found that most of the students' learning space was located in their own bedroom, and the desk was placed in a corner of the bedroom. In order to better simulate the students' learning space, the desk of the laboratory was also placed in a corner of the room. The desk lamp is placed on the left side of the working surface and the light outlet is about $500 \mathrm{~mm}$ away from the working surface. This placement distance is the best distance [13].

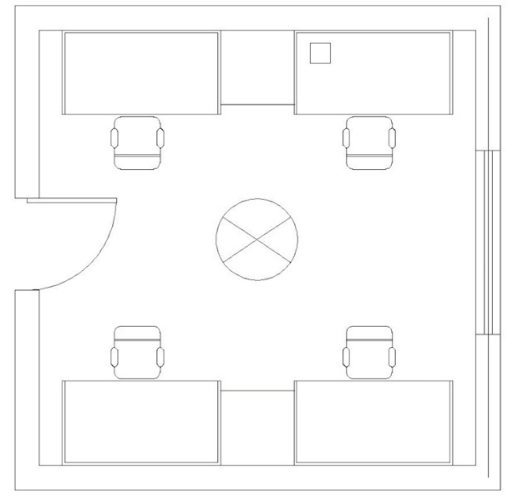

Fig.1 Experimental laboratory
Philips $32 \mathrm{~W}$ annular lamp with a color temperature of $4000 \mathrm{~K}$ is used as the background lighting for the laboratory. The local lighting is a Panasonic LED lamp. The color temperature range of the desk lamp covers three grades: low, medium and high. The illuminance range is $0-15001 x$, and the light is evenly distributed. The experiment uses the SFIM-400 spectral scintillation illuminometer to adjust and test the illuminance of the working surface.

When exploring the influence of illuminance on eyestrain and visual efficacy. Medium color temperature (4500k), which is most favorable for relieving eyestrain and improving visual efficacy [14], is selected for the experiment. The lighting method is mixed lighting. In this experiment, the background lighting and color temperature are kept unchanged, and the illuminance of the working face is changed by adjusting the desk lamp. According to "Architectural Lighting Design Standards" GB 50034-2013[12]: The minimum variation of subjective perceived illuminance is about 1.5 times, so the illuminance in the experiment is selected from five levels of 3001x, 5001x, 7501x, 10001x and 15001x.

When exploring the effect of color temperature on eyestrain and visual efficacy. In the experiment, the illuminance of 5001x, which is conducive to improving visual efficacy [15], is selected for the experiment. The lighting method is mixed lighting, and the experiment is carried out by changing the color temperature of the desk lamp. At present, some scholars have researched the influence of lighting environment on eyestrain and visual efficacy by focusing on color temperature, and three different levels of color temperature have been selected as experimental variables. Therefore, in the experiment, low color temperature $3500 \mathrm{k}$, medium color temperature $4500 \mathrm{k}$ and high color temperature $6000 \mathrm{k}$ were also selected for research.

When exploring the effects of lighting methods on eyestrain and visual efficacy, the experiments on the influence of illumination intensity on eye fatigue and visual efficacy under mixed lighting conditions were compared. In the experiment, only the lighting method was changed to local lighting, the color temperature remained unchanged, and the illuminance was set of six levels: 1501x, 3001x, 5001x, 7501x, 10001x and 15001x. A new set of low-illuminance experiments with an illuminance of $1501 \mathrm{x}$ was added to the experiment. The reason is that after the background lighting was removed, the desk lamp was adjusted to carry out the experiment with a low-illumination limit value, so that the results were more clearly compared.

\subsubsection{Evaluation method}

Eyestrain evaluation

The near-point measurement method can be used to evaluate the subjects' eye accommodation before and after the experiment, and to measure the degree of eyestrain. During the test, the end of the accommodation convergence rule was placed at the tip of the subject's nose, and the icon moved slowly and uniformly toward the end of the ruler. When the subject had blurred vision, stopped 
moving and recorded the current reading on the ruler. The larger the reading, the greater the distance of the nearpoint, that is, the more serious the eyestrain. The optimal lighting environment is determined by comparing the degree of eyestrain under different lighting environments. The calculation method of the degree of eyestrain is as follows:

$$
\varepsilon=\left|d_{a}-d_{b}\right| \div d_{b}
$$

In formula (1), $\varepsilon$ represents the degree of eyestrain; $d_{a}$ represents the near point distance after learning; $d_{b}$ represents the near point distance before learning.

Visual efficacy evaluation

Dose-work test is a commonly used method of measuring visual efficacy. Changes in visual efficacy of subjects can be measured by completing a given amount of work. The questionnaire in Anfimov test was used in this experiment, which has 8 different letters, namely A, B, C, E, H, K, N, X. There are 1200 letters in the table, each of which appears with the same frequency, and the letter height in the table is $3 \mathrm{~mm}$. At the beginning, the subjects were instructed to delete a letter within a certain period of time. During the process of completing the questionnaire, the subjects should read from left to right without skipping lines. The letters to be deleted in the instruction for each questionnaire cannot be the same. The experiment evaluated visual efficacy by calculating the rate of change of the index of the subjects' mental work capacity (IMC), the larger the change rate of IMC, the better the visual efficacy. The calculation method is as follows:

$$
\eta=\left(n_{r} \div 2\right) \times\left[\left(n_{c}-n_{m}\right) \div n_{c}\right]
$$

In formula (2), $\eta$ represents the index of mental work efficacy (IMC); $n_{r}$ represents the number of letters to read; $n_{c}$ represents the number of letters correctly deleted; $n_{m}$ represents the number of letters deleted by mistake.

$$
\mu=\left|\eta_{\mathrm{a}}-\eta_{b}\right| \div \eta_{b}
$$

In formula (3), $\mu$ represents the change rate of IMC,

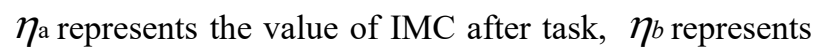
the value of IMC before task.

\subsubsection{Experimental method}

During the experiment, first of all, the purpose and specific methods of the experiment were explained to the subjects, so that the experimenters were familiar with the experiment process. Then, the lighting environment was adjusted for the three experiments on the influence of illuminance, color temperature and lighting method on eyestrain and visual efficacy, and the subjects were tested with the same method. The specific steps are as follows: Subjects should be acquainted with the questionnaire in Anfimov test and complete the task for at least 5 times. Meanwhile, they should be familiar with the accommodation convergence rule to ensure the follow-up eye accommodation test. After that, the eyes of subjects were dropped with eyedrops to relieve eyestrain and rested for three minutes, keeping their eyes relaxed. In the experiment, first of all, the questionnaire in Anfimov test and the eye accommodation test were carried out. After completion, the subjects worked on the working surface under the lighting conditions required by the experiment. The work materials are all junior high school homework, and the work time is 45 minutes. After the work is completed, the questionnaire in Anfimov test and the eye accommodation test will be carried out again.

Further calculation of the experimental results were used to determine the eyestrain and the change rate of IMC, and to measure the effects of illuminance, color temperature, and lighting method on eyestrain and visual efficacy.

\section{Results}

\subsection{Effect of illuminance on eyestrain and visual efficacy}

When the background lighting and color temperature (4500k) remain unchanged, only the illuminance of the local lighting is changed, and the changes in the mean value of the degree of eyestrain and the mean value of IMC under the five illuminance conditions of 3001x, 5001x, 7501x, 10001x and 15001x are obtained, as shown in Fig.2.

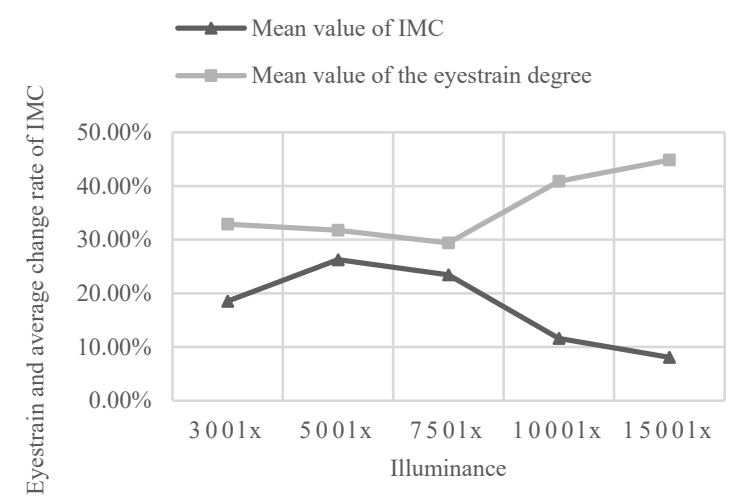

Fig.2 Effect of illuminance on eyestrain and visual efficacy

It can be seen that the degree of eyestrain first decreases with the increase of illuminance. When the illuminance is $7501 \mathrm{x}$, the degree of eyestrain reaches the lowest value of $29.41 \%$. When the illuminance is greater than $7501 x$, the degree of eyestrain increases with the increase of illuminance. In general, when the illuminance is 3001x and 5001x, the degree of eyestrain is lower than $35 \%$, which is close to that of $7501 x$. However, when the illuminance is $10001 \mathrm{x}$, the degree of eyestrain is more than $40 \%$, and when the illuminance is $15001 \mathrm{x}$, the degree of eyestrain is the highest.

In the IMC change rate curve, the change rates of IMC are all positive under the five illumination conditions, indicating that all the five illuminance levels could improve the visual efficacy of the subjects. With the increase of illuminance, the change rate of IMC first increased and then decreased. When the illuminance is $5001 x$, the changing rate reaches the maximum value of $26.28 \%$, followed by $7501 x$.

Therefore, the degree of eyestrain is lowest when the illuminance is $7501 \mathrm{x}$, and Illuminance at 5001x is most beneficial for improving visual efficiency. When the illuminance is 5001x and 7501x, the degrees of eyestrain 
are all lower than $35 \%$, and the improvements of visual efficacy are also higher than $20 \%$. It is believed that the illuminance of $5001 \mathrm{x}-7501 \mathrm{x}$ is beneficial to relieving eyestrain and improving visual efficacy.

\subsection{Effect of color temperature on eyestrain and visual efficacy}

When the lighting mode and illuminance (5001x) remain unchanged, only the color temperature is changed, and the changes in the mean value of the degree of eyestrain and the mean value of IMC under the three color temperature conditions of $3000 \mathrm{k}, 4500 \mathrm{k}, 6000 \mathrm{k}$ are obtained, as shown in Fig.3.

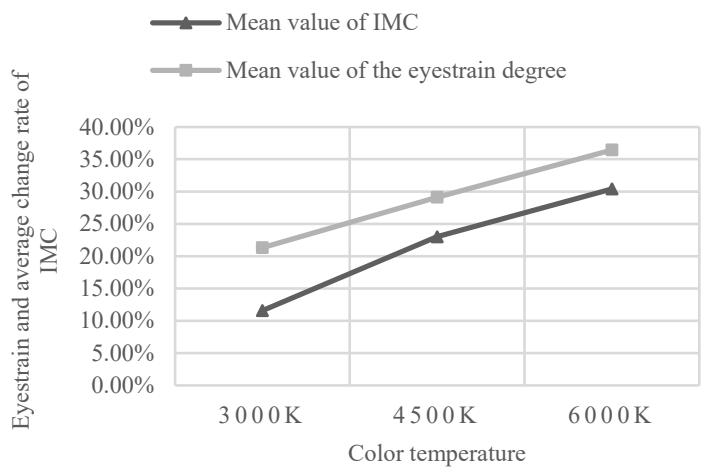

Fig.3 Effect of color temperature on eyestrain and visual efficacy

It can be seen that the color temperature and the degree of eyestrain are positively correlated. As the color temperature increases, the degree of eyestrain also increases. When the color temperature is $3000 \mathrm{k}$, the degree of eyestrain is the lowest at $21.33 \%$. As the color temperature increases to $6000 \mathrm{k}$, the degree of eyestrain also increases to $36.44 \%$. Thus, in terms of relieving eyestrain, a low color temperature of $3000 \mathrm{k}$ is better.

The change rates of IMC in the figure 3 are all positive, indicating that all three color temperatures can improve the visual efficacy of the subjects. At the same time, there is a positive correlation between color temperature and the change rate of IMC. The change rate of IMC increased with the increase of color temperature.

Therefore, a low color temperature of $3000 \mathrm{k}$ is conducive to relieving eyestrain, while a high color temperature of $6000 \mathrm{k}$ is conducive to improving visual efficacy.

\subsection{Effect of lighting method on eyestrain and visual efficacy}

When keeping the color temperature $(4500 \mathrm{k})$ unchanged and turning off the background lighting, the changes in the mean value of the degree of eyestrain under six illuminance conditions of 1501x, 3001x, 5001x, 7501x, 10001x and 15001x is obtained, as shown in Fig.4.

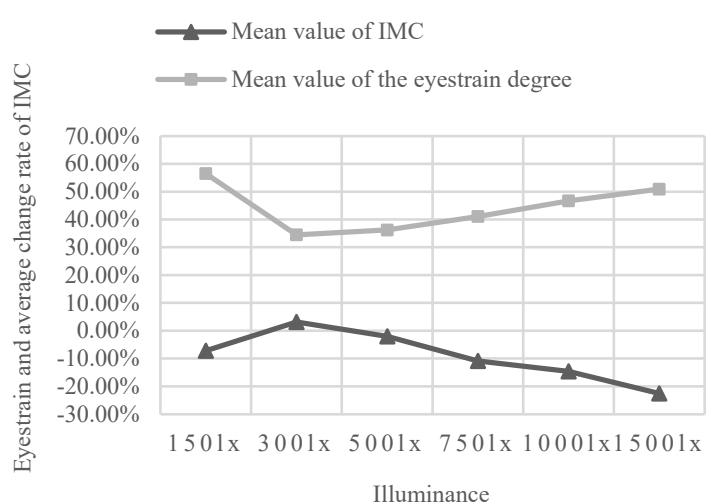

Fig.4 Effect of lighting mode on eyestrain and visual efficacy

It can be seen that the degree of eyestrain decreases first and then increases with the increase of illuminance, reaching the lowest value of $34.49 \%$ at $3001 x$, among which the degree of eyestrain reaches the highest at 1501x, which may be caused by low illuminance. In terms of the change rate of IMC, it is positive only when the illuminance is $3001 \mathrm{x}$, and the other parameter values are negative, indicating that under local lighting, the illuminance is $3001 \mathrm{x}$, and has a slight effect on improving the visual efficiency, while under other illuminance conditions, the visual efficiency decreases.

By comparing the experimental data under the condition of background lighting, the results are shown in Fig. 5 and 6.

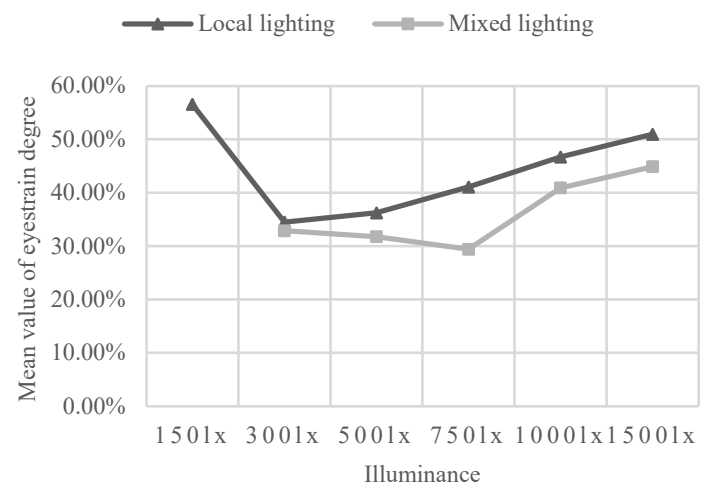

Fig.5 The mean value of the degree of eyestrain

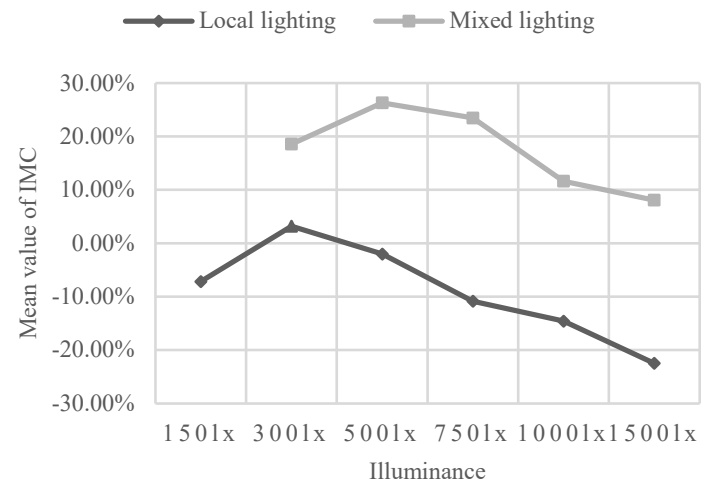

Fig.6 The average change rate of IMC 
It can be seen that the degree of eyestrain under local lighting conditions are all higher than that under mixed lighting conditions. In terms of visual effects, mixed lighting is also more conducive to improving visual effects than local lighting. Hence, compared with local lighting, the mixed lighting effect is better.

Therefore, in the process of designing the lighting environment of the home learning space, a mixed lighting method is preferred, and it is advantageous to select the illuminance from 5001x to 7501x. When there is only local lighting, the illuminance can be appropriately reduced to relieve eyestrain and improve visual efficiency.

\section{Conclusion}

At present, the number of teenagers with myopia in our country ranks first in the world, and the Myopia rate of teenager is increasing year by year. The eye health problem of students has become a serious challenge. At the same time, due to the increase in the amount of homework for middle school students, the study time at home becomes longer, and the lighting environment of home night learning space is particularly important for students' eye health. In this paper, 112 home learning Spaces were measured, users' satisfaction with lighting environmental parameters was investigated, and experiments were designed to explore the effects of illuminance, color temperature and lighting mode on eyestrain and visual efficacy. The experimental results show that under the condition of mixed lighting, when the color temperature $(4500 \mathrm{k})$ remains unchanged, the illuminance in the range of 5001x to 7501x is beneficial to relieving eyestrain and improving the visual efficacy; When the illuminance (5001x) remains unchanged, low color temperature $(3500 \mathrm{k})$ helps to relieve eyestrain, and high color temperature $(6000 \mathrm{k})$ can improve the visual efficacy better. In addition, the eye strain of mixed lighting is lower than that of local lighting, and the visual efficiency is much higher than that of lighting methods (only local lighting). The conclusions drawn from the study have certain reference significance for the reasonable improvement of the home lighting environment.

\section{Acknowledgments}

The study has been supported by the China National Key R\&D Program "Research on the energy efficiency and health performance improvement of building operations based on lifecycle carbon emissions reduction" (Grant No. 2018YFE0106100).

\section{References}

1. World Health Organization. Blindness and vision impairment. https://www.who.int/zh/news-room/factsheets/detail/blindness-and-visual-impairment. (2021)

2. Morgan IG, Ohno-Matsui K, Saw SM. Myopia. Lancet. 379(9827):1739-1748 (2012)

3. $\mathrm{Wu}$, Pei-Chang $\mathrm{MD}, \mathrm{PhD}$, et al. Epidemiology of
Myopia, Asia-Pacific Journal of Ophthalmology. 5(6): 386-393 (2016)

4. Gong Chunzi, Zhang Honglin, Enumerate the "vision" of teenagers. J. China Statistics. (12):62-64 (2020)

5. Guo Zhen, Xie Sen, Du Xianli, et al. Analysis of influencing factors of screening myopia among junior high school students in six provinces in china. J. Chinese Journal of School Health. 41 (11): 1703$1706(2020)$

6. Xiao H, Cai H, Li X. Non-visual effects of indoor light environment on humans: A review. J. Physiology \& Behavior. 228:113195 (2020)

7. De Giuli, Valeria; Da Pos, Osvaldo; De Carli, Michele. (2012)Indoor environmental quality and pupil perception in Italian primary schools. Building and Environment. (56): 335- 345

8. Zadnik K, et al. Prediction of Juvenile-Onset Myopia. JAMA Ophthalmol.133(6):683-9 (2015)

9. Morgan I, Rose K. How genetic is school myopia? Prog Retin Eye Res. 24(1):1-38 (2005)

10. Shimomura $\mathrm{Y}$, Iwanaga $\mathrm{K}$, Harada $\mathrm{H}$, et al. Effects of the Color Temperature of Illuminance on Arousal Level in Working. J. J Physiol Anthropol. 20(2):149 (2001)

11. Inoue $M$, katsuura $T$, Harada $H$. The Effect of Color Temperature on Visual Fatigue. J. J Physiol Anthropol Appl Human Sci. 17 (5):218 (1997)

12. Standard for lighting design of buildings (GB500342013), China Architecture \& Building Press. Beijing, China. (2013)

13. Chen Chaozhong, Shi Xiaohong, Wang Ye, et al. (2012) Re-Discussing about the "Eye-Protecting Table Lamp". J. China Illuminating Engineering Journal. 23 (6): 59-63

14. Niu Pingjuan, Fang Jinglu, Tian Huijuan, et al. Study on the Influence of LED Office Lighting Environment Based on Photobiological Effect. J. China Illuminating Engineering Journal. 000 (004): 23-28, 34 (2014)

15. Goven T, Laike T, Raynham P, et al. The influence of ambient lighting on pupils in classroom considering visual, biological and emotional aspects as well as use of energy. C. Proceedings of the International Commission on Illumination Conference. Vienna. Austria. (2010) 